\title{
Constraints on the Environment Depence of the Neutrino Mass from Solar and Reactor Neutrino Data
}

\author{
Pedro Cunha de Holanda \\ Instituto de Física, Universidade de São Paulo, Cidade Universitária, 05508-900, São Paulo, SP, Brazil
}

Received on 18 July, 2006

\begin{abstract}
We investigate what can be the role of Mass Varying Neutrinos in several different neutrino oscillation experiments, in particular focusing on the relation between KamLAND data and solar neutrino experiment results. Assuming that the adiabaticity of solar neutrinos evolution in LMA-MSW scenario is not broken by the inclusion of this new mechanism, we can establish new limits on MaVaN's parameters by fitting together KamLAND and solar neutrinos data. We also investigate the role of how non-adiabatic effects in the Sun can change this scenario, allowing a larger value of these parameters.
\end{abstract}

Keywords: Neutrino oscillations; Solar neutrino puzzle

\section{INTRODUCTION}

In this article we investigate the characteristic effects of the dependence of the neutrino mass on the visible matter environment for solar and reactor neutrinos. We find that, although the inclusion of the environment dependent terms can lead to certain improvement of the quality of the fit in the most favour LMA region for well determined values of the new parameters, (in agreement with the result of Ref. [1]), this improvement does not hold much statistical significance. However, the inclusion of these effects, can substantially improve the quality of the fit in the high- $\Delta m^{2}$ (LMA-II) region which can be allowed at $98.9 \%$ CL. Still, if we assume that the presence of this new effect is not strong enough to break adiabaticity of solar neutrino evolution, the combined analysis of the solar [2-7] and KamLAND data [8] in these scenarios results into a constraint on the possible dependence of the neutrino mass on the ordinary matter density. Consequently, we derive an upper bound on the product of the effective neutrino-scalar $\left(\lambda^{v}\right)$ and matter-scalar $\left(\lambda^{f}\right)$ Yukawa couplings, which depends on the mass of the scalar field.

We investigate also the possibility that this new mechanism plays a leading role in reactor and solar neutrinos instead of the usual conversion mechanism through mass and mixing. In this context, reactor neutrino data are fully explained by a mass-varying neutrino mechanism, and strong non-adiabatic effects in solar neutrino evolution produces the correct energy dependence in solar neutrino conversion to reproduce experimental data.

\section{FORMALISM}

The formalism adopted here follow the one in [9], where an effective low energy model containing the Standard Model particles plus a light scalar $(\phi)$ of mass $m_{S}$ couples very weakly both to neutrinos $\left(v_{i}\right)$ and the matter fields $f=e, n, p$.

The Lagrangian takes the form

$$
\mathcal{L}=\sum_{i} \bar{v}_{i}\left(i \not \partial-m_{i}^{0}\right) v_{i}+\sum_{f} \bar{f}\left(i \not \partial-m_{f}^{0}\right) f+\frac{1}{2}\left[\phi\left(\partial^{2}-m_{S}^{2}\right) \phi\right]+\sum_{i j} \lambda_{i j}^{v} \bar{v}_{i} v_{j} \phi+\sum_{f} \lambda^{f} \bar{f} f \phi
$$

where $m_{i}^{0}$ are the vacuum mass neutrinos would have in the presence of the cosmic neutrino background, $\lambda_{i j}^{v}$ and $\lambda^{f}$ are, respectively, the effective neutrino-scalar and matter-scalar couplings. We have written a Lagrangian for Dirac neutrinos but equivalently it could be written for Majorana neutrinos.
In a medium with some additional neutrino background (either relativistic or non-relativistic) as well as non-relativistic matter (electrons, protons and neutrons), neutrinos acquire masses which obey the following set of integral equations

$$
\begin{aligned}
m_{i j}(r) & =m_{i}^{0} \delta_{i j}-M_{i j}(r) \\
M_{i j}(r) & =\frac{\lambda_{i j}^{v}}{m_{S}^{2}}\left(\sum_{f} \lambda^{f} n_{f}(r)+\sum_{a} \lambda^{v}{ }_{a a} \int \frac{d^{3} k}{(2 \pi)^{3}} \frac{M_{a a}}{\sqrt{k^{2}+M_{a a}^{2}}} f_{a}(r, k)\right),
\end{aligned}
$$


where $n_{f}(r)$ is the number density for the fermion $f$, and $f_{a}(r, k)$ is the sum of neutrino and antineutrino " $a$ " occupation numbers for momentum $k$ in addition to the cosmic background neutrinos.

We neglect the contribution to the neutrino mass from the background neutrino density and we concentrate on the matter density dependence:

$$
M_{i j}(r)=\frac{\lambda_{i j}^{v}}{m_{S}^{2}} \sum_{f} \lambda^{f} n_{f}(r)
$$

This is very similar to the scenario considered in Ref.[1].

\section{EFFECTS IN SOLAR NEUTRINO OSCILLATIONS}

The minimum joint description of atmospheric [10], $\mathrm{K} 2 \mathrm{~K}$ [11], solar [2-7] and reactor [8, 12] data requires that all the three known neutrinos take part in the oscillations. The mixing parameters are encoded in the $3 \times 3$ lepton mixing matrix which can be conveniently parametrized in the standard form

$$
U=\left(\begin{array}{ccc}
1 & 0 & 0 \\
0 & c_{23} & s_{23} \\
0 & -s_{23} & c_{23}
\end{array}\right)\left(\begin{array}{ccc}
c_{13} & 0 & s_{13} e^{i \delta} \\
0 & 1 & 0 \\
-s_{13} e^{-i \delta} & 0 & c_{13}
\end{array}\right)\left(\begin{array}{ccc}
c_{21} & s_{12} & 0 \\
-s_{12} & c_{12} & 0 \\
0 & 0 & 1
\end{array}\right)
$$

where $c_{i j} \equiv \cos \theta_{i j}$ and $s_{i j} \equiv \sin \theta_{i j}$.

According to the current data, the neutrino mass squared differences can be chosen so that

$$
\Delta m_{\odot}^{2}=\Delta m_{21}^{2} \ll\left|\Delta m_{31}^{2}\right| \simeq\left|\Delta m_{32}^{2}\right|=\Delta m_{\mathrm{atm}}^{2} .
$$

As a consequence of the fact that $\Delta m_{21}^{2} /\left|\Delta m_{31}^{2}\right| \approx 0.03$, for solar and KamLAND neutrinos, the oscillations with the atmospheric oscillation length are completely averaged and the interpretation of these data in the neutrino oscillation framework depends mostly on $\Delta m_{21}^{2}, \theta_{12}$ and $\theta_{13}$, while atmospheric and $\mathrm{K} 2 \mathrm{~K}$ neutrinos oscillations are controlled by $\Delta m_{31}^{2}, \theta_{23}$ and $\theta_{13}$. Furthermore, the negative results from the $\mathrm{CHOOZ}$ reactor experiment [12] imply that the mixing angle connecting the solar and atmospheric oscillation channels, $\theta_{13}$, is severely constrained $\left(\sin ^{2} \theta_{13} \leq 0.041\right.$ at $3 \sigma$ [13]). Altogether, it is found that the $3-v$ oscillations effectively factorizing into $2-v$ oscillations of the two different subsystems: solar and atmospheric.

With the inclusion of the environment dependence terms (Eq. (3)) it is not warranted that such factorization holds. We will assume that this is still the case and study their effect on solar and KamLAND oscillations under the hypothesis of one mass-scale dominance. Under this assumption, we work in two different parametrization of the evolution equation, depending of what is the main conversion mechanism, as explained in next sessions.

\section{MSW-LMA + MAVAN'S AS A SUBLEADING EFFECT}

Assuming MaVaN's is a subleading effect in solar and reactor neutrino's conversion, we use the following parametrization, as in [1]:

$$
i \frac{d}{d r}\left(\begin{array}{c}
\mathrm{v}_{e} \\
\mathrm{v}_{\mu}
\end{array}\right)=\left[\frac{1}{2 E_{\mathrm{v}}} \mathbf{U}_{\theta_{12}}\left(\begin{array}{cc}
M_{1}^{2}(r) & M_{3}^{2}(r) \\
M_{3}^{2}(r) & \left(m_{2}^{0}-M_{2}(r)\right)^{2}
\end{array}\right) \mathbf{U}_{\theta_{12}}^{\dagger}+\left(\begin{array}{cc}
V_{\mathrm{CC}}(r) & 0 \\
0 & 0
\end{array}\right)\right]\left(\begin{array}{l}
\mathrm{v}_{e} \\
v_{\mu}
\end{array}\right)
$$

where we have assumed the $m_{1}^{0}=0$. Here $V_{\mathrm{CC}}(r)=$ $\sqrt{2} G_{F} n_{e}(r)$ is the MSW potential proportional to the electron number density $n_{e}(r)$ in the medium. $\mathbf{U}_{\theta_{12}}$ is the $2 \times 2$ mixing matrix in vacuum parameterized by the angle $\theta_{12}$, and $M_{i}(r)$ are the environment dependent kinetic contributions to the neutrino masses.

In general, for given matter density profiles, Eq. (6) has to be solved numerically. As discussed in Ref.[1] in most of parameter space allowed by KamLAND and solar data, for all practical purposes, the transition is adiabatic and the evolution equation can be solved analytically to give the survival probability

$$
P_{e e}=\frac{1}{2}+\frac{1}{2} \cos 2 \theta_{0,12}^{m} \cos 2 \theta_{12}
$$

where $\theta_{0,12}^{m}$ is the effective neutrino mixing angle at the neutrino production point $r_{0}$ in the medium, explicitly given by

$$
\cos 2 \theta_{0,12}^{m}=\frac{\left(\Delta \tilde{M}_{21}^{2}\left(r_{0}\right) \cos 2 \tilde{\theta}_{0,12}-2 E_{v} V_{\mathrm{CC}}\left(r_{0}\right)\right)}{\sqrt{\left(\Delta \tilde{M}_{21}^{2}\left(r_{0}\right) \cos 2 \tilde{\theta}_{0,12}-2 E_{\mathrm{v}} V_{\mathrm{CC}}\left(r_{0}\right)\right)^{2}+\left(\Delta \tilde{M}_{21}^{2}\left(r_{0}\right) \sin 2 \tilde{\theta}_{0,12}\right)^{2}}}
$$


with

$$
\begin{array}{r}
\Delta \tilde{M}_{21}^{2}\left(r_{0}\right)=2 \sqrt{M_{3}^{4}\left(r_{0}\right)+\left(\frac{\Delta M_{21}^{2}\left(r_{0}\right)}{2}\right)^{2}} \\
\cos 2 \tilde{\theta}_{0,12}=\frac{\frac{\Delta M_{21}^{2}\left(r_{0}\right)}{2} \cos 2 \theta_{21}-M_{3}^{2}\left(r_{0}\right) \sin 2 \theta_{12}}{\sqrt{M_{3}^{4}\left(r_{0}\right)+\left(\frac{\Delta M_{21}^{2}\left(r_{0}\right)}{2}\right)^{2}}}
\end{array}
$$

and where

$$
\Delta M_{21}^{2}\left(r_{0}\right)=\left(m_{2}^{0}-M_{2}\left(r_{0}\right)\right)^{2}-M_{1}^{2}\left(r_{0}\right) .
$$

In general, $M_{i}(r)$ can be an arbitrary function of the background matter density. For the sake of concreteness we will assume a dependence in accordance with the results obtained in the linear approximation given in Eq.(3). So we will parametrize these terms as:

$$
M_{i}(r)=\alpha_{i}\left[\frac{\rho(r)}{\left(\mathrm{gr} / \mathrm{cm}^{3}\right)}\right],
$$

where $\rho$ is the matter density, and from Eq.(3) we find the characteristic value of the $\alpha$ coefficients to be

$$
\alpha \sim 4.8 \times 10^{23} \lambda^{v} \lambda^{N}\left(\frac{10^{-7} \mathrm{eV}}{m_{S}}\right)^{2} \mathrm{eV} .
$$

Most precisely, in its simplest realization, Eq. (3) implies that $M_{3}^{2}(r)$ would not only depend on $\rho^{2}(r)$ but it would also adquire a contribution proportional to $\rho(r)$. In what follows we have neglected this linear contribution but we have verified that its presence would not modify our conclusions.

One must notice, however, that, as long as the transition is adiabatic, the survival probability only depends on the value of $M_{i}(r)$ at the neutrino production point. Therefore it only depends on the exact functional form of $M_{i}(r)$ via the averaging over the neutrino production point distributions.

The survival probability for anti-neutrinos, $P_{\bar{e} \bar{e}}$, which is relevant for KamLAND, takes the form

$$
P_{\bar{e} \bar{e}}=1-\sin ^{2} 2 \tilde{\theta}_{0,12}^{m} \sin ^{2}\left(\frac{\Delta m_{K L}^{2} L}{2 E_{\mathrm{V}}}\right),
$$

where $\cos 2 \tilde{\theta}_{0,12}^{m}$ is defined as in Eq. (8) and $\Delta m_{K L}^{2}$ is the denominator of this equation but replacing $V_{\mathrm{CC}}$ by $-V_{\mathrm{CC}}$ and assuming a constant matter density $\rho \sim 3 \mathrm{gr} / \mathrm{cm}^{3}$, typical of the Earth's crust.

\section{A. Constraints from Solar and Reactor Neutrino Data}

We present in this section the results of the global analysis of solar and KamLAND data with environment dependent neutrinos masses for the specific realization discussed in the previous section. Furthermore, for simplicity, we will restrict ourselves to the case $M_{1}(r)=m_{1}=0$.
Details of our solar neutrino analysis have been described in previous papers $[14,15]$. We use the solar fluxes from Bahcall and Serenelli (2005) [16]. The solar neutrino data includes a total of 119 data points: the Gallium [3, 4] and Chlorine [2] (1 data point) radiochemical rates, the Super-Kamiokande [5] zenith spectrum (44 bins), and SNO data reported for phase 1 and phase 2. The SNO data used consists of the total daynight spectrum measured in the pure $\mathrm{D}_{2} \mathrm{O}$ (SNO-I) phase (34 data points) [6], plus the full data set corresponding to the Salt Phase (SNO-II) [7]. This last one includes the NC and ES event rates during the day and during the night (4 data points), and the $\mathrm{CC}$ day-night spectral data (34 data points). In combining with the SNO-I data, only the theoretical uncertainties are assumed to be correlated between the two phases. The experimental systematics errors are considered to be uncorrelated between both phases.

For KamLAND we directly adapt the $\chi^{2}$ map as given by the KamLAND collaboration for their unbinned rate+shape analysis [17] which uses 258 observed neutrino candidate events and gives, for the standard oscillation analysis, a $\chi_{\min }^{2}=701.35$. The corresponding Baker-Cousins $\chi^{2}$ for the 13 energy bin analysis is $\chi_{\min }^{2}=13.1 / 11$ dof. The effect of MaVaN's parameters in KamLAND result was calculated assuming a constant Earth density of $3 \mathrm{~g} / \mathrm{cm}^{3}$, and assuming that KamLAND are sensitive to the vacuum value of $\Delta m_{0,21}^{2}$ and $\theta_{12}$ through an effective mass and mixing in a constant Earth density, given by Eqs. (9) and (10) as described in Eq. (14).

In presence of the environment dependence contribution to the masses, the analysis of solar and KamLAND data depends on four parameters: the two standard oscillation parameters $\Delta m_{0,21}^{2}=\left(m_{2}^{0}\right)^{2}$, and $\tan ^{2} \theta_{12}$, and the two environmentdependence coefficients, $\alpha_{2}$, and $\alpha_{3}$. In this case, in order to cover the full CP conserving parameter space we allow the $\alpha$ parameters to vary in the range

$$
-\infty \leq \alpha_{2} \leq \infty \quad-\infty \leq \alpha_{3}^{2} \leq \infty
$$

We find the best fit point

$$
\begin{array}{cc}
\tan ^{2} \theta_{12}=0.49 & \Delta m_{0,21}^{2}=8.4 \times 10^{-5} \mathrm{eV}^{2} \\
\alpha_{2}=10^{-4} \mathrm{eV} & \alpha_{3}=i 2.0 \times 10^{-5} \mathrm{eV} .
\end{array}
$$

This is to be compared with the best fit point for no envi- 
ronment dependence of the neutrino mass $\alpha_{2}=\alpha_{3}=0$

$$
\begin{gathered}
\tan ^{2} \theta_{12}=0.44 \quad \Delta m_{0,21}^{2}=7.9 \times 10^{-5} \mathrm{eV}^{2} . \\
\Delta \chi^{2}=2.5
\end{gathered}
$$

where $\Delta \chi^{2}$ is given with respect to the minimum in Eq.(16). Thus we find that although the inclusion of the environment dependent terms can lead to a small improvement of the quality of the fit (in agreement with the result of Ref. [1]), this improvement is not statistically very significant leading only to a decrease of $\Delta \chi^{2}=2.5$ even at the cost of introducing two new parameters.

We show in Fig. 1 the result of the global analysis of solar data plus KamLAND data in the form of the allowed twodimensional regions at $3 \sigma \mathrm{CL}$ in the $\left(\Delta m_{21,0}^{2}, \tan ^{2} \theta_{12}\right)$ plane after marginalization over $\alpha_{2}$ and $\alpha_{3}$. The standard MSW allowed region is also showed for reference. As seen in the figure, allowing for environment dependence of the neutrino masses enlarges only slightly the allowed range of $\Delta m_{21,0}^{2}$ and $\tan ^{2} \theta_{12}$. In contrast to the standard MSW analysis, where the limits on the mixing angle come basically from solar neutrinos, here it is KamLAND data that control the lower limits for the mixing angle.

Most interestingly, we also find that the description of the solar data in the high- $\Delta m^{2}$ (LMA-II) region can be significantly improved so there is a new allowed solution at the 98.9\% CL. The best fit point in this region is obtained for

$$
\begin{aligned}
\tan ^{2} \theta_{12}=0.5 & \Delta m_{0,21}^{2}=1.75 \times 10^{-4} \mathrm{eV}^{2} \\
\alpha_{2}=1.3 \times 10^{-4} \mathrm{eV} & \alpha_{3}=i 2.0 \times 10^{-5} \mathrm{eV} \\
\Delta \chi^{2}=8.9 &
\end{aligned}
$$

While this region is excluded at more than $4 \sigma$ for standard MSW oscillations, it can appear at less $3 \sigma$ in the presence of environmental effects with $\left|\alpha_{3}\right| \leq 3.2 \times 10^{-5}$ and $2.8 \times 10^{-5} \leq \alpha_{2} \leq 2.0 \times 10^{-4}$. Basically this region appears at the CL which is presently allowed by KamLAND data [8] because the fit to the solar data, once the possibility of environment dependence is included, cannot discriminate between the LMA-I and LMA-II regions. Clearly this implies that this solution will be further tested by a more precise determination of the antineutrino spectrum in KamLAND.

Conversely the global analysis of solar and KamLAND data results into the constraint of the possible size of the environment dependent contribution to the neutrino mass. This is illustrated in Fig. 2 where we show the result of the global analysis in the form of the allowed two-dimensional regions in the $\left(\alpha_{2}, \alpha_{3}\right)$ parameter space after marginalization over $\Delta m_{0,21}^{2}, \tan ^{2} \theta_{12}$. The full regions correspond to $1 \sigma, 95 \%$ and $3 \sigma \mathrm{CL}$ while the curves correspond to 90 and $99 \% \mathrm{CL}$. As seen in the figure, for $\mathrm{CL}>1.1 \sigma$ the regions are connected to the $\alpha_{2}=\alpha_{3}=0$ case and they are always bounded. In other words, the analysis show no evidence of any environment dependence contribution to the neutrino mass and there is an upper bound on the absolute values of the corresponding coefficients.

In order to quantify the bound on MaVaN's parameters, we proceed a marginalization over the parameters $\Delta m_{0,21}^{2}, \tan ^{2} \theta_{12}$ and $\alpha_{3}\left(\alpha_{2}\right)$, obtaining the following $90 \% \mathrm{CL}(3 \sigma)$, bounds (with 1dof).

$$
\begin{aligned}
-2.2 \times 10^{-5} \leq \alpha_{2} / \mathrm{eV} \leq 1.4 \times 10^{-4} & \left(-5.6 \times 10^{-5} \leq \alpha_{2} / \mathrm{eV} \leq 1.7 \times 10^{-4}\right) \\
\left|\alpha_{3}\right| / \mathrm{eV} \leq 2.3 \times 10^{-5} & \left(\left|\alpha_{3}\right| / \mathrm{eV} \leq 8.4 \times 10^{-5}\right) \text { for } \alpha_{3}^{2}>0 \\
\left|\alpha_{3}\right| / \mathrm{eV} \leq 3.4 \times 10^{-5} & \left(\left|\alpha_{3}\right| / \mathrm{eV} \leq 5.2 \times 10^{-5}\right) \text { for } \alpha_{3}^{2}<0
\end{aligned}
$$

These bounds can be converted into a bound on the product of the characteristic effective neutrino-scalar and matterscalar couplings which must be accounted for when building specific models of MaVaNs. For example, at 90\% CL,

$$
\left|\lambda^{v} \lambda^{N}\right|\left(\frac{10^{-7} \mathrm{eV}}{m_{S}}\right)^{2} \leq 3.0 \times 10^{-28}
$$

\section{MAVANS AS THE LEADING CONVERSION MECHANISM}

More generically non-adiabatic effects occur for sufficiently large values of the $\alpha$ parameters so that one can disregard the standard MSW potential $V_{\mathrm{CC}}$ and the vacuum mass $m_{2}^{0}$ with respect to the matter density mass dependent terms.
In this case, as seen from Eq. (8), the mixing angle inside the Sun is constant and controlled by the $\alpha^{\prime} s$. At the border of the its vacuum value in a strongly non-adiabatic transition. In this context the MaVaN's effects will be crucial also to interpretate the reactor neutrinos data, not anymore as a subleading effect, but as the main leading flavor conversion mechanism.

We parametrize the new MaVaN's term slightly different from last section, having for the evolution matrix:

$$
H=\frac{1}{2 E} H_{M V N}+\frac{1}{2 E} \mathbf{U}_{\theta_{12}}\left(\begin{array}{cc}
0 & 0 \\
0 & m_{2}^{2}
\end{array}\right) O \mathbf{U}_{\theta_{12}}^{\dagger}+\left(\begin{array}{cc}
V_{C C} & 0 \\
0 & 0
\end{array}\right)(23)
$$

where each term in $H_{M V N}$ has the same squared dependence on baryonic density, $H_{M V N}^{(i, j)}=\alpha_{i j} \rho^{2}$. Sun, as the density goes to zero, the mixing angle is driven to 


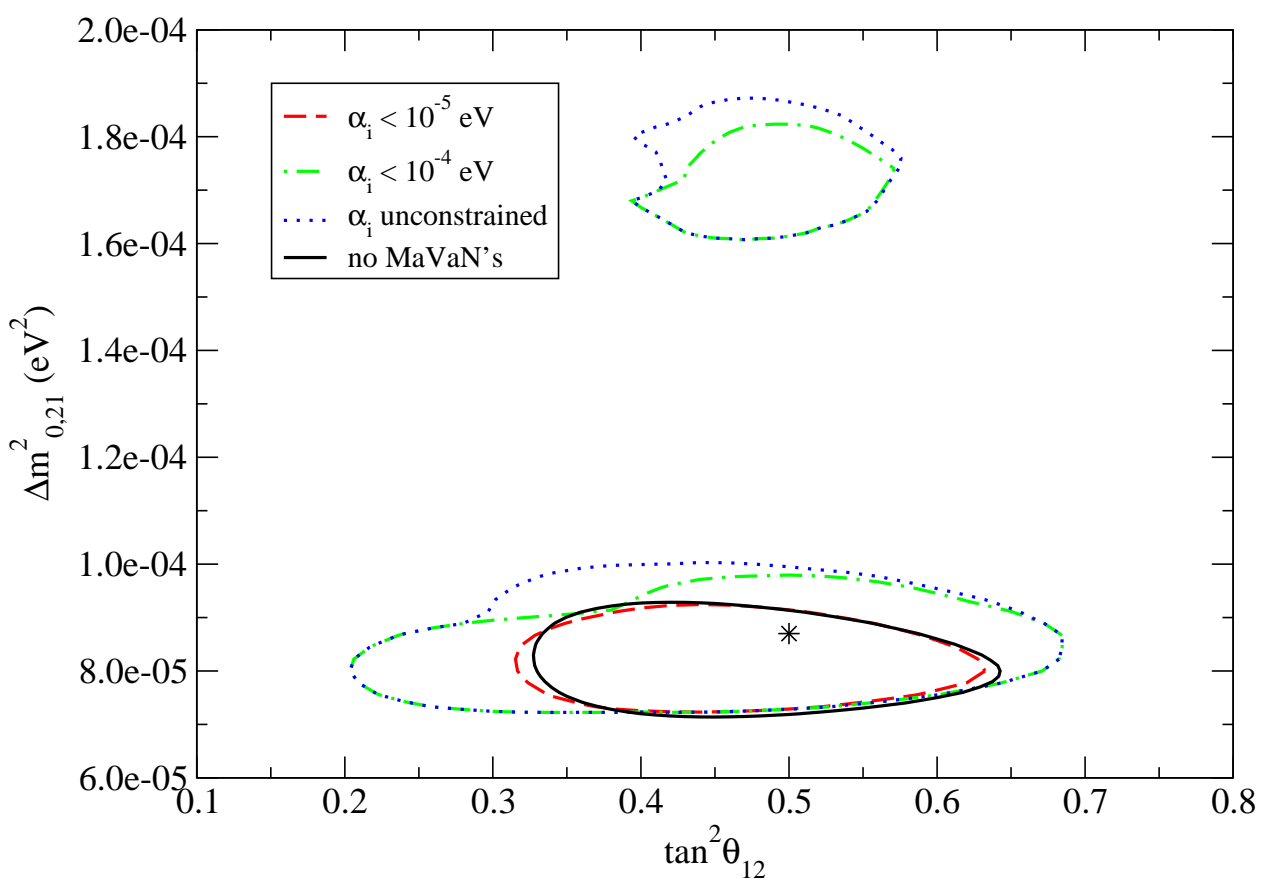

FIG. 1: Allowed regions from the global analysis of solar plus KamLAND data in the $\left(\Delta m_{0,21}^{2}, \tan ^{2} \theta_{12}\right)$ parameter space at $3 \sigma$ CL (2dof). The best fit point at $\tan ^{2} \theta_{12}=0.5$ and $\Delta m_{0,21}^{2}=8.7 \times 10^{-5} \mathrm{eV}^{2}$ is represented by the star. The standard MSW allowed region is also shown for reference.

We will work in a context where reactor neutrino oscillations are caused exclusively by MaVaN's effects, and vacuum oscillation is effective only in vacuum, so $\alpha_{i, j} \rho_{\text {mantle }}^{2}>>$ $V_{C C}>>H_{\text {vac }}^{i, j}$.

For neutrinos who crosses the Earth mantle, the MaVaN's term dominates over both vacuum terms and standard matter interactions. We can determine each one of its entries using data from neutrino oscillation experiments who crosses the Earth:

$$
\Delta \tilde{m}_{21}^{2}=8 \times 10^{-5} \mathrm{eV}^{2} \quad ; \quad \tan ^{2} \tilde{\theta}_{12}=0.5
$$

so we can write:

$$
H_{M V N}=\frac{1}{2 E} \mathbf{U}_{M V N}\left(\begin{array}{cc}
0 & 0 \\
0 & \Delta \tilde{m}_{21}^{2}
\end{array}\right) O_{M V N}^{\dagger} \times\left(\frac{\rho}{\rho_{\text {mantle }}}\right)^{2}
$$

where

$$
\mathbf{U}_{M V N}=\left(\begin{array}{cc}
\tilde{c}_{12} & \tilde{s}_{12} \\
-\tilde{s}_{12} & \tilde{c}_{12}
\end{array}\right)
$$

As said before, at the center of the Sun the MaVaN's term dominates over both vacuum and standard matter interaction terms, so the evolution hamiltonian is given by eq. (25), with mixing angles given by eq. (24). When neutrino aproaches the Sun surface, the density decreases as $\rho^{2}$, and at some point the matter term $V_{C C}$ starts to be important.

There is a point where $V_{C C}=\frac{\Delta \tilde{m}}{2 E} \cos 2 \tilde{\theta}_{12}$ and a resonance between two first families is produced, with $\theta_{12}=\pi / 4$. From this point this mixing angle continues to grow, achieving the value of $\theta_{12}=\pi / 2$ when the $V_{C C}$ becomes predominant over the MaVaN's terms. At the border of the Sun (but still inside it) the neutrino flux will be an energy dependent admixture of $v_{1}$ and $v_{2}$, with no production of $v_{3}$, and with mixing angles $\theta_{12}=\pi / 2$ and $\theta_{23}=\pi / 4$. Preliminar calculations show that an intersting spectral distortion on electron neutrino survival probability can be produced by such mechanism.

One important point of this mechanism is that it naturally produces a strong day-night asymmetry in solar neutrino fluxes, due to the strong interaction with Earth's baryonic matter. This can be avoided by some tuning in usual neutrino's mass and mixings in a three generation scenario. A detailed analysis of the possibility to produce a general fit to solar neutrino data will be presented elsewhere [18].

\section{DISCUSSION}

We have investigated the phenomenological consequences of a scalar induced environment dependence of the effective neutrino mass in the interpretation of solar and reactor neutrino data.

For the sake of concreteness, we consider an effective low energy model containing the Standard Model particles plus a light scalar $(\phi)$ of mass $m_{S}$ which couples very weakly both to neutrinos $\left(\mathrm{v}_{i}\right)$ and the matter fields $f=e, n, p$ which can induce a dependence of the neutrino mass on the density of the medium. This is described in Sec. II and its consequences to neutrino oscillations in the Sun is discussed in Sec. III.

We have performed a combined analysis of the solar neutrino data (118 data points) and KamLAND (17 data points) 

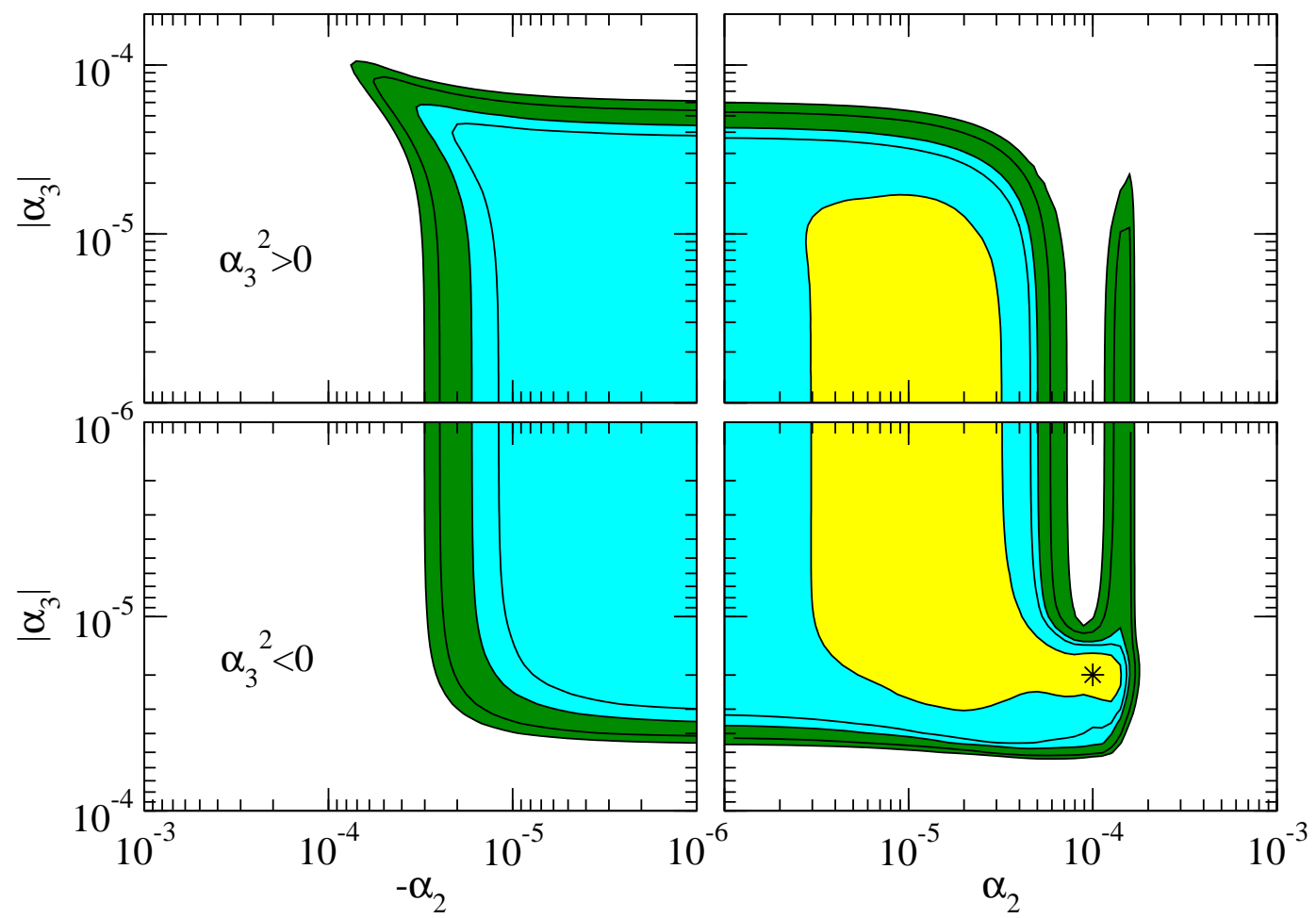

FIG. 2: Allowed regions from the global analysis of solar and solar plus KamLAND data in the $\left(\alpha_{2}, \alpha_{3}\right)$ parameter space. The curves correspond to $1 \sigma, 90 \%, 95 \%, 99 \%$ and $3 \sigma \mathrm{CL}$ (2dof). The best fit point at $\alpha_{2}=10^{-4} \mathrm{eV}$ and $\alpha_{3}=i 2.0 \times 10^{-5} \mathrm{eV}$, represented by a star, is also shown.

in the context of this effective model. Our analysis, which is described in Sec. IV A, depends on 4 parameters: the two standard oscillation parameters $\Delta m_{0,21}^{2}=\left(m_{2}^{0}\right)^{2}$, and $\tan ^{2} \theta_{12}$, and the two environment-dependence coefficients, $\alpha_{2}$, and $\alpha_{3}$. We found the best fit point at: $\tan ^{2} \theta_{12}=0.49, \Delta m_{0,21}^{2}=$ $8.4 \times 10^{-5} \mathrm{eV}^{2}, \alpha_{2}=10^{-4} \mathrm{eV}$ and $\alpha_{3}=i 2.0 \times 10^{-5} \mathrm{eV}$. This point corresponds to a decrease of $\Delta \chi_{\min }^{2}=-2.5$ in comparison to the minimum in the case where no environment dependence is considered. We conclude that in spite of the inclusion the two extra parameters, there is no a very statistically significant improvement of the quality of the fit in the most favoured LMA-I MSW region.

We find that the description of the solar data in the high$\Delta m^{2}$ (LMA-II) region can be significantly improved so there is a new allowed solution at the $98.9 \% \mathrm{CL}$. The best fit point in this region is obtained for $\tan ^{2} \theta_{12}=0.5, \Delta m_{0,21}^{2}=1.75 \times$ $10^{-4} \mathrm{eV}^{2}, \alpha_{2}=1.3 \times 10^{-4} \mathrm{eV}$ and $\alpha_{3}=i 2.0 \times 10^{-5} \mathrm{eV}$. This solution will be further tested by a more precise determination of the antineutrino spectrum in KamLAND.
In any case, our data analysis permit us to considerably limit the size of the $\alpha$ coefficients (see Eq. (21)) and from that derive a limit on the product of the effective neutrino-scalar and matter-scalar Yukawa couplings depending on the mass of the scalar field (Eq. (22)). These constrains have to be taken into account when constructing specific models of MaVaNs. These scenarios will be further tested by the precise determination of the energy dependence of the survival probability of solar neutrinos, in particular for low energies [19].

We also are investigating the possible role of MaVaN's effect as a leading mechanism in neutrino flavor conversion. This work is in progress, and will be further detailed in next communications.

\section{Acknowledgments}

This work was supported by Fundação de Amparo à Pesquisa do Estado de São Paulo (FAPESP).
[1] V. Barger, P. Huber and D. Marfatia, [arXiv:hep-ph/0502196.]

[2] B.T. Cleveland et al., Astrophys. J. 496 (1998) 505.

[3] C. Cattadori, Results from radiochemical solar neutrino experiments, talk at XXIst International Conference on Neutrino Physics and Astrophysics (NU2004), Paris, June 14-19, 2004.

[4] GALLEX collaboration, Phys. Lett. B 447 (1999) 127.
[5] Super-Kamiokande Collaboration, S. Fukuda et al., Phys. Rev. Lett. 86 (2001) 5651

[6] SNO Collaboration, Q.R. Ahmad et al., Phys. Rev. Lett. 87 (2001) 071301; Phys. Rev. Lett. 89 (2002); Phys. Rev. Lett. 89 (2002) 011302; SNO Collaboration, S.N. Ahmed et al., Phys. Rev. Lett. 92 (2004), 181301. 
[7] SNO Collaboration, S B. Aharmim et al., nucl-ex/0502021.

[8] KamLAND collaboration, K. Eguchi et al., Phys. Rev. Lett. 94 (2005) 081801; Phys. Rev. Lett. 90 (2003) 021802.

[9] M. C. Gonzalez-Garcia, P. C. de Holanda, and R. Zukanovich Funchal, Phys. Rev. D 73, 033008 (2006).

[10] Y. Ashie et al. [Super-Kamiokande Collaboration], Phys. Rev. D 71, 112005 (2005).

[11] E. Aliu et al. [K2K Collaboration], Phys. Rev. Lett. 94, 081802 (2005).

[12] CHOOZ Collaboration, M. Apollonio et al., Phys. Lett. B 420 , 397 (1998); Eur. Phys. J. C 27, 331 (2003).

[13] M. C. Gonzalez-Garcia, arXiv:hep-ph/0410030.

[14] J.N. Bahcall, M.C. Gonzalez-Garcia, and C. Peña-Garay, JHEP 02 (2003) 009 [hep-ph/0212147]; J.N. Bahcall and C. Peña-
Garay, JHEP. 11 (2003) 004.

[15] P. C. de Holanda and A. Y. Smirnov, Astropart. Phys. 21, 287 (2004).

[16] J. N. Bahcall and A. M. Serenelli, Astrophys. J. 626, 530 (2005).

[17] KamLAND data and $\chi^{2}$ results are available at http://www.awa.tohoku.ac.jp/KamLAND/datarelease/2ndresult.html

[18] M. C. Gonzalez-Garcia, P. C. de Holanda, M. Maltoni, and R. Zukanovich Funchal, in preparation.

[19] Low Energy Solar Neutrino Detection (LowNu2), ed. by Y. Suzuki, M. Nakahata, and S. Moriyama, World Scientific, River Edge, NJ, 2001. 